\title{
Combined Topo-Guided laser Vision Correction (LASIK) with Concurrent Prophylactic High-Fluence Corneal Collagen Crosslinking (CXL) in Myopic Patients with Normal Cornea \\ Khaled EL-Ghonemy Said Ahmed, Hany Ahmed Khiry, Ahmed Ibrahim Basiony, Rana Mohamed Amer Elwakiel* \\ Department of Ophthalmology, Faculty of Medicine, Menoufia University, Egypt
}

*Corresponding author: Rana Mohamed Amer Elwakiel, Mobile: (+20) 01110518599, E-Mail: ronielwakiel@gmail.com

\begin{abstract}
Background: Corneal ectasia is a serious vision-threatening complication of laser-assisted in-situ keratomileusis (LASIK). LASIK in combination with corneal collagen cross-linking (CXL) is an alternative to traditional LASIK aimed at restoring strength to the cornea.

Objective: The aim of the work was to evaluate the efficacy and safety of ultraviolet A (UVA) irradiation crosslinking in concurrent with LASIK in myopic patient with normal cornea to avoid post LASIK ectasia.

Patients and Methods: This Prospective randomized interventional study included 60 eyes of 30 consecutive patient who were scheduled for LASIK surgery, all of them were myopic with normal cornea with no pervious refractive surgery. They were candidate for Combined Topo-Guided LASIK with accelerated CXL. This was done in a private eye hospital between Jun 2018 and Jun 2019.

Results: The preoperative uncorrected visual acuity (UCVA) ranged from $0.05-0.1$ with mean $0.075 \pm 0.02$ and preoperative best corrected visual acuity (BCVA) ranged $0.2-1.0$ with mean $0.58 \pm 0.13$ and post-operative UCVA at 6 months showed ranged 0.2-1.0 with mean 0.55 \pm 0.16 , and postoperative UCVA at 1 year showed ranged 0.2-1.0 with mean $0.56 \pm 0.13$,. The improvement in visual acuity from $0.075 \pm 0.02$ UCVA preoperatively show statistically significant difference $(\mathrm{P}>0.05)$ because we compared the results with the preoperative BCVA $0.58 \pm 0.13$ postoperatively at 6 months. However, t-Paired Sample t-test; p-value < $0.05 \mathrm{~S}$.

Conclusion: It could be concluded that LASIK combined with a prophylactic CXL intervention appears to provide predictability as well as refractive and keratometric stability. The data reported in this study provide evidence of the safety and efficacy of this approach.
\end{abstract}

Keywords: LASIK, CXL, Myopic Patients, Normal Cornea

\section{INTRODUCTION}

Laser assisted in situ keratomileusis (LASIK) is a widely accepted method to correct refractive errors and offers predictable and stable refractive and visual outcomes, specifically in the correction of moderate to high myopia (equal to or greater than -6.00 diopters).The principle of LASIK is to ablate corneal stroma by laser technique, altering central corneal curvature to correct refractive error and focus incident light on the retina ${ }^{(\mathbf{1})}$.

Postoperative ectasia is a result of corneal alteration by severing of stromal lamellae through the creation of the LASIK flap, combined with stromal ablation, which together can weaken corneal biomechanical properties and affect the corneal stability. The application aims to enhance corneal rigidity and thus reduce the likelihood of a long-term myopic shift ${ }^{(2)}$.

The corneal collagen cross-linking (CXL) procedure increases covalent bonding between or within collagen fibers in the corneal stroma, thus increasing corneal stiffness and improving corneal biomechanical stability. The long-term efficacy of CXL has already been demonstrated in the treatment of primary and secondary (post-LASIK and post-pRK) corneal ectasia ${ }^{(3)}$.
LASIK in combination with CXL (Lasik Xtra Avedro, Massachusetts, USA) is an alternative to traditional LASIK aimed at restoring strength to the cornea, increasing stability in visual outcomes, increasing the accuracy of the refractive correction, and the structural integrity of the cornea. It is logical to anticipate that stiffening a cornea, which has been structurally weakened by LASIK through the addition of CXL may minimize the negative effects associated with this biomechanical compromise, so the aim of Lasik Xtra is to further reduce the rare incidence of iatrogenic ectasia, as well as to reduce the rate of hypermetric treatment regression and enhancements ${ }^{(4)}$.

The objective of this study was to evaluate the efficacy and safety of UVA irradiation crosslinking in concurrent with LASIK in myopic patient with normal cornea to avoid post LASIK ectasia.

\section{PATIENTS AND METHODS}

This prospective randomized interventional study included a total of 60 eyes of 30 consecutive patient who were scheduled for LASIK surgery, attending at private Eye Hospital. This study was conducted between Jun 2018 and Jun 2019. 


\section{Ethical approval:}

Written informed consent of all the subjects for intervention including advantages and disadvantages, risk of possible complication and periodical follow up for 6 months after surgery was obtained. Approval of the ethical committee of Menoufia University was obtained.

All patients were myopic with normal cornea and with no pervious refractive surgery. They were subjected to Combined Topo-Guided LASIK with accelerated CXL.

Inclusion criteria: Included age $\geq 19$ years, preoperative spherical refractive error from -1.00 -D to $-10.00 \mathrm{D}$ spherical error, cylinder $-1.0 \mathrm{D}$ to $-5.00 \mathrm{D}$, and stable refractive error.

Exclusion criteria: Included central corneal thickness (CCT) less than 500um, predicted postoperative residual stroma bed thickness of less than $\mathbf{3 0 0 \mu \mathrm { m }}$, Participants were instructed to remove soft contact lenses at least 2 weeks before the screening examination.

1. History of previous refractive surgery.

2. History of viral corneal ulcer or keratitis.

3. Age less than 18 years.

4. Corneal dystrophy, scars, irregular astigmatism and corneal ectatic diseases.

5. History of corneal or intraocular surgery, glaucoma \& retinal detachment.

6. Patients with congenital abnormalities (iris, Pupil deformation, etc.).

7. Macular degeneration or retinopathy and Neuroophthalmic diseases.

All the patients underwent history and complete ophthalmological examination including:

1. Uncorrected visual acuity and best-corrected visual.

2. Slit-lamp examination of the cornea, anterior, posterior segment of the eye and Intraocular pressure measurement

3. Fundus examination with peripheral retinal exam for any retinal tear.

4. Ocular history taking of constant eye rubbing and Family history of KC.

5. Corneal topography \& Pachymetry.

6. Study will be subjected to the following examination preoperatively and follow-up visits at 1,3 , and 6 months.

\section{Operative Procedure Technique:}

Before the patient had entered the surgery room, the eye lids were washed After topical anesthesia with Benox ED (Fluorescein-Benoxinate, EIPICO ), the patient made comfortable, taking care to position his head correctly so that the body was aligned with his head ${ }^{(5)}$.
Allegretto wave " Eye -Q Eximer Laser, all corneas were subjected to myopic LASIK treatment with Flaps $(90 \mu \mathrm{m}$ thick) were created with the Wave Light excimer laser (Alcon). A lid speculum was placed to provide a maximum exposure of the eye to avoid interference with the placement of the suction ring and the excursion of the microkeratome head. The suction ring was placed, the vacuum activated the microkeratome was placed on the suction ring, the path was checked to assure no obstacles, and the microkeratome cut was made, Microkeratome used was Moria One Use Disposable. A blunt spatula was used to reflect the flap upon its hinge then stromal bed was dried with a LASIK sponge.

The Soaking time was 1 min then excess riboflavin was wiped from the cornea surface, the flap stroked back into place and the interface was irrigated under oblique illumination to assure that all debris were removed. Special care was taken to minimize potential riboflavin soaking to the folded LASIK flap. We used part $2-0.22 \%$ riboflavin solution Avedro without dextran was instilled on the exposed stromal bed. Ultraviolet A (UVA) exposure as the flap was in contact with the riboflavin-soaked stroma, as riboflavinpresoaked flap participating strongly in the UVA absorption.

\section{Post-operative follow-up:}

In early post-operative period, all patients were treated by co-avazier eye drops (Tobramycin $0.3 \%$ (3 $\mathrm{mg} / \mathrm{ml})$, Dexamethasone $0.1 \%(1 \mathrm{mg} / \mathrm{ml})$ ) Preservative: benzalkonium chloride mg Orchidia ) five times per day and tapered gradually over five weeks, topical antibiotics eye drops five times per day and lubricant Hyfresh eye drops (Sodium Hyaluronate $2.0 \mathrm{mg}$. Jamjoom ) five times per day.

There was post-operative follow up visits within 18 months: 1 day, 1 week, 2 weeks, 1 month, 2 months, 6 months, 9 months, 12 months, 18 months postoperatively.

\section{Statistical analysis}

Recorded data were analyzed using the statistical package for social sciences, version 20.0 (SPSS Inc., Chicago, Illinois, USA). Quantitative data were expressed as mean \pm standard deviation (SD). Qualitative data were expressed as frequency and percentage. Paired sample t-test of significance was used when comparing between related sample. Independent-samples t-test of significance was used when comparing between two means. The confidence interval was set to $95 \%$ and the margin of error accepted was set to 5\%. So, the p-value was considered significant as the following: P-value $<0.05$ was considered significant. P-value $<0.001$ was considered as highly significant. P-value $>0.05$ was considered insignificant. 


\section{RESULTS}

60 eyes of 30 consecutive patient who were scheduled for LASIK surgery, there were 8 male ( mean $26.7 \%$ ) and 22 female ( mean $73.3 \%$ ). Their ages ranged from 21-33 with mean age (27.05 \pm 4.71$)$. Follow up period from 12-18 months with mean 12.11 months. All patients were myopic with normal cornea with no pervious refractive surgery. They were candidate for Combined Topo-Guided LASIK with accelerated CXL this was done in a private eye hospital. between Jun 2018 and Jun 2019.

Table (1): Demographic data distribution of the study group.

\begin{tabular}{|l|c|}
\hline Demographic data & Total $(\mathbf{n}=\mathbf{6 0})$ \\
\hline Gender & $8(26.7 \%)$ \\
Male & $22(73.3 \%)$ \\
Female & \\
\hline Age (years) & $27.05 \pm 4.71$ \\
Mean \pm SD & $38(63.3 \%)$ \\
\hline & $22(36.7 \%)$ \\
\hline 30 years & years
\end{tabular}

This table shows that the male (26.7\%) and female (73.3\%) of gender, while ranged aged 21-33 with mean $27.05 \pm 4.71$

Table (2): Comparison between Pre-operative UCVA and Post-operative BCVA.

\begin{tabular}{|l|l|l|l|l|}
\hline & Mean \pm SD & Mean Diff. & t-test & p-value \\
\hline Pre-operative UCVA & $0.075 \pm 0.02$ & \multirow{2}{*}{0.505} & \multirow{2}{*}{3.681} & \multirow{2}{*}{$0.003^{*}$} \\
\hline Pre-operative BCVA & $0.58 \pm 0.13$ & & & \\
\hline
\end{tabular}

t-Paired Sample t-test; *p-value $<0.05 \mathrm{~S}$

This table shows statistically significant difference between Pre-operative and Pre-operative UCVA.

Table (3): Comparison between Post-operative UCVA at 6m and Post-operative UCVA at 1year.

\begin{tabular}{|l|l|l|l|l|}
\hline & Mean \pm SD & Mean Diff. & t-test & p-value \\
\hline Post-operative UCVA at $6 \mathrm{~m}$ & $0.55 \pm 0.16$ & 0.001 & 0.097 & \multirow{2}{*}{0.773} \\
\hline Post-operative UCVA at 1year & $0.56 \pm 0.13$ & & & \\
\hline
\end{tabular}

t-Paired Sample t-test; p-value >0.05 NS

This table shows no statistically significant difference between post-operative UCVA at $6 \mathrm{~m}$ and postoperative UCVA at 1 year.

Table (4): Comparison between Pre-operative and Post-operative refraction.

\begin{tabular}{|l|l|l|l|l|}
\hline Refraction & Mean \pm SD & Mean Diff. & t-test & p-value \\
\hline Sphere Pre & $-5.03 \pm 2.46$ & 4.880 & -16.232 & $<0.001^{* *}$ \\
\hline Sphere Post at 6m & $-0.15 \pm 0.36$ & & & \\
\hline Sphere Post at 1 year & $-0.14 \pm 0.34$ & 2.300 & -12.579 & $<0.001^{* *}$ \\
\hline Cylinder Pre & $-2.36 \pm 1.00$ & & & \\
\hline Cylinder Post at 6m & $-0.06 \pm 0.74$ & & 2.186 & \multirow{2}{*}{$0.038^{*}$} \\
\hline Cylinder Post at 1 year & $-0.06 \pm 0.65$ & -17.950 & & \\
\hline Axial Pre & $113.95 \pm 56.24$ & & & \\
\hline Axial Post at 6m & $96.00 \pm 62.15$ & & & \\
\hline Axial Post at 1 year & $93.41 \pm 51.62$ & & & \\
\hline
\end{tabular}

t-Paired Sample t-test; p-value >0.05 NS; *p-value <0.05 S; **p-value <0.001 HS

This table shows statistically significant difference between Pre-operative and Post-operative according to their refraction regarding sphere, cylinder and axial, while post at $6 \mathrm{~m}$ insignificant comparing post at 1 year with $\mathrm{p}$-value $>0.05 \mathrm{NS}$. 
Table (5): Comparison between Pre-operative and Post-operative according to pachymetry.

\begin{tabular}{|l|l|l|l|c|}
\hline Pachymetry & Mean \pm SD & Mean Diff. & t-test & p-value \\
\cline { 1 - 2 } Pre-operative & $531.20 \pm 22.56$ & \multirow{2}{*}{-99.450} & \multirow{2}{*}{38.798} & \multirow{2}{*}{$<0.001^{* *}$} \\
\hline Post-operative at $6 \mathrm{~m}$ & $431.75 \pm 31.20$ & 4.57 & 0.761 & 0.317 \\
\hline Post-operative at 1 year & $427.18 \pm 25.48$ & & \\
\hline
\end{tabular}

t-Paired Sample t-test; $* *$ p-value $<0.001 \mathrm{HS}$

This table shows highly statistically significant difference between Pre-operative and Post-operative according to pachymetry, while post at $6 \mathrm{~m}$ insignificant comparing post at 1 year with p-value $>0.05$ NS.

Table (6): Comparison between Pre-operative and Post-operative according to K reading.

\begin{tabular}{|c|c|c|c|c|}
\hline k reading & Mean \pm SD & Mean Diff. & t-test & p-value \\
\hline K1 Pre & $42.16 \pm 0.93$ & \multirow{2}{*}{-4.250} & \multirow{2}{*}{17.411} & \multirow{2}{*}{$<0.001 * *$} \\
\hline $\mathrm{K} 1$ Post at $6 \mathrm{~m}$ & $37.91 \pm 1.62$ & & & \\
\hline K1 Post at 1 year & $36.78 \pm 1.34$ & 1.13 & 1.019 & 0.519 \\
\hline K2 Pre & $44.32 \pm 1.01$ & \multirow{2}{*}{-5.620} & \multirow{2}{*}{39.248} & \multirow{2}{*}{$<0.001 * *$} \\
\hline $\mathrm{K} 2$ Post at $6 \mathrm{~m}$ & $38.70 \pm 1.66$ & & & \\
\hline K2 Post at 1 year & $37.85 \pm 1.21$ & 0.85 & 0.796 & 0.356 \\
\hline
\end{tabular}

t-Paired Sample t-test; $\quad$ p-value $>0.05 \mathrm{NS} ; *$ p-value $<0.05 \mathrm{~S} ; * *$ p-value $<0.001 \mathrm{HS}$

This table shows statistically significant difference between Pre-operative and Post-operative according to their $\mathrm{K}$ reading regarding $\mathrm{K} 1$ and $\mathrm{K} 2$, while post at $6 \mathrm{~m}$ insignificant comparing post at 1 year with $\mathrm{p}$-value $>0.05 \mathrm{NS}$.

\section{DISCUSSION}

The current study revealed CXL with Combined Topo-Guided laser Vision Correction (LASIK) including 60 myopic eyes with normal cornea demonstrated that this protocol appeared safe and efficient. The objective of this study was to evaluate the efficacy and safety of UVA irradiation crosslinking in concurrent with LASIK in myopic patient with normal cornea to avoid post LASIK ectasia.

This study included 30 patients. Their ages ranged from 21-33 years with mean age (27.05 \pm 4.71$)$. This result is in agreement with that of Kanellopoulos et al. ${ }^{(6)}$ who selected patients aged 19 to 39 years, with a mean of $27.5 \pm 6.1$ years. However, their preoperative central corneal thickness ranged from 474 to $595 \mu \mathrm{m}$ with a mean of $545.96 \pm 33.93 \mu \mathrm{m}$, whereas our patients' corneal thickness ranged from 501 to $580 \mu \mathrm{m}$ with mean $531.20 \pm 22.56$ also median (532) of corneal pachymetry and post-operative central corneal thickness ranged from 395 - to $518 \mu \mathrm{m}$

We carried out LASIK correction of myopic patients with the application of CXL in the protocol mentioned before in patients and methods. the preoperative UCVA ranged from 0.05- 0.1 with mean $0.075 \pm 0.02$ and preoperative BCVA ranged 0.2- 1.0 with mean $0.58 \pm 0.13$ and post-operative UCVA at 6 months showed ranged 0.2-1.0 with mean 0.55 \pm 0.16 , and postoperative UCVA at 1 year showed ranged $0.2-$ 1.0 with mean $0.56 \pm 0.13$, The improvement in visual acuity from $0.075 \pm 0.02$ UCVA preoperatively show statistically significant difference $(\mathrm{P}>0.05)$ because we compared the results with the preoperative BCVA $0.58 \pm 0.13$ postoperatively at 6 months. However, tPaired Sample t-test; $\mathrm{p}$-value $<0.05 \mathrm{~S}$. P-value $>0.05$ NS shows no statistically significant difference between post-operative UCVA at $6 \mathrm{~m}$ and postoperative UCVA at 1year.

A study done by Reinstein et al. ${ }^{(7)}$ showed no statistically significant differences in UDVA and CDVA between the two groups from preoperative to post-operative except in the UDVA $(\mathrm{P}<0.001)$ and CDVA $(\mathrm{P}=0.005)$ at 1 month after operation. All eyes had emmetropia as the target refraction. At 6 months after operation, the efficacy index (mean postoperative UDVA/mean preoperative CDVA) was $1.02 \pm 0.18$ in LASIK-ACXL group and $1.06 \pm 0.25$ in the LASIK-only group. Shows the UDVA of LASIK-ACXL group and LASIK-only group at 6 months after operation.

In previous retrospective study by

Kanellopoulos et $\boldsymbol{a l} .{ }^{(8)}$ which was a comparable to our study reported on 43 patients treated with femtosecond laser flap and the Wave Light excimer platform with a UVA irradiation power of $10 \mathrm{~mW} / \mathrm{cm} 2$ for $3 \mathrm{~min}$. This method appeared to be a safe and effective adjunctive treatment for refractive regression and potential ectasia. The mean UCVA changed from 0.2 to 1.2 , BCVA from 1.1 to $1.2, \mathrm{SE}$ from -7.5 to $-0.2 \mathrm{D}$ to $1.2, \mathrm{BCVA}$ from 1.1 to $1.2, \mathrm{SE}$ from -7.5 to $-0.2 \mathrm{D}$, and keratometry from 44.5 to 38 
D. None of the cases developed signs of ectasia or significant regression during a mean follow-up duration of 3.5 years. Kanellopoulos (said that this application could be viewed as a 'prophylactic customization of the biomechanical behavior of corneal collagen further combined operation using the same parameter are applied in a contralateral eye study of 34 consecutive patients reached the same conclusion. Eyes that underwent CXL demonstrated mean regression from treatment of $+0.22 \pm 0.31 \mathrm{D}$, whereas eyes that did not undergo CXL showed a greater regression of $+0.72 \pm 0.19 \mathrm{D}$ at 2 years postoperatively. In the research of Kanellopoulos and Pamel ${ }^{(9)}, 27$ of 32 eyes had an improvement in UDVA and CDVA of $20 / 45$ or better (2.25 log MAR) (8).

Regarding postoperative complication, no significant changes were detected after 1 year follow up, a study by Alessio et al. (10) including 17 patients (34 eyes) who underwent PRK followed by CXL versus CXL alone suggested that the combined procedure was better in reducing corneal aberrations and stabilizing progressive keratoconus. The changes in UDVA were from $0.63 \pm 0.36$ to $0.19 \pm 0.17 \mathrm{log}$ MAR and from $0.59 \pm 0.29$ to $0.52 \pm 0.29 \log$ MAR, respectively.

Regarding postoperative residual manifest refraction, no significant errors were detected after 1 year follow up. A study by Celik et al. ${ }^{(11)}$ four patients underwent LASIK with concurrent accelerated CXL in one eye and LASIK only in the fellow eye to treat myopia or myopic astigmatism. During the surgery $0.1 \%$ riboflavin solution was instilled into the corneal bed for $1.5 \mathrm{~min}$ while the flap remained open, and UVA exposure was performed for $3 \mathrm{~min}$ at a power of $30 \mathrm{~mW} / \mathrm{cm} 2$ after flap closure. They attempted correction (SE) ranged from -5.00 to $-8.50 \mathrm{D}$ in the LASIK-CXL group and from -3.00 to -7.25 in the LASIK- only group. The LASIK-CXL group had a UDVA and manifest refraction equal to or better than those in the LASIK- only group.

The incidence of postoperative corneal ectasia among studies, there were no cases after 1 year follow-up presented with corneal ectasia, Kymionis et al. ${ }^{(12)}$ reported a simultaneous combination operation may also be used to treat corneal ectasia after LASIK. The (UDVA) and (CDVA) showed significant improvements from 20/100 to 20/40 and from 20/40 to $20 / 25$, respectively.

Reinstein et al. ${ }^{(13)}$ found that rapid CXL had minimal impact on the overall corneal stiffness after LASIK flap creation at any stromal depth. Using the previously established log-log relationship could calculate the mechanical impact of the various procedures analyzed in terms of traditional moduli. Their comparison of flap-cut and virgin corneas showed that the Brillouin shifts significantly decreased by approximately $35 \mathrm{MHz}$, corresponding to a Young's modulus reduction of approximately $26 \%$ after the LASIK flap creation.

Regarding flap thickness, there were no cases presented with failed flap creation in our study flap was done ranged 90-100 with mean 90.702 \pm 2.577 , also median (90) and interquartile range (0) of flap thickness. Similarly, in study by Reinstein et al. ${ }^{\text {(7) }}$ detected Biomechanical Changes with Brillouin Microscop. Their Results showed a total of 11 eyes were evaluated by Brillouin imaging and Brillouin depth profile measurements in the virgin state, after LASIK flap creation, and after rapid CXL, which revealed the varying elastic modulus of cornea throughout the depth consistent with previous measurements. Brillouin shifts were highest, corresponding to highest corneal strength, After LASIK flap creation the Brillouin shifts in the anterior and central regions showed a marked reduction. comparison of Brillouin shifts in flap-cut corneas compared to virgin corneas .showed statistically significant decrease of the Brillouin shifts, denoting a statistically significant weakening due to LASIK flap creation, Although the Brillouin shift was slightly higher after rapid CXL, there was no statistically significant difference in strength at any level of the corneas.

Regarding hyperopic regression, although our study is only for myopic patient but it was proved to be very significant for hyperopic regression, In a study by Aslanides and Mukherjee (14) that reported the long- term outcomes, safety, stability, and efficacy of a pilot series of simultaneous LASIK and CXL with an illumination of $3 \mathrm{~mW} / \mathrm{cm} 2$ for $15 \mathrm{~min}$, the average $\mathrm{SE}$ was $+3.4 \mathrm{D}$ after 4 years, the authors suggested that this technique may be promising for ameliorating hyperopic regression. This study also found that keratometry decreased from $42.91 \pm 2.01 \mathrm{D}(\mathrm{K} 1)$ preoperatively to $39.65 \pm 2.91 \mathrm{D}$ on the second day postoperatively and flattened to $39.61 \pm 2.74$, $39.65 \pm 2.73$, and $39.48 \pm 2.68 \mathrm{D}$ at 1,3 , and 6 months postoperatively, respectively, and preoperative $\mathrm{K} 2$ was $44.37 \pm 2.29 \mathrm{D}$ and flattened to $41.06 \pm 3.22$, $40.56 \pm 3.12,40.54 \pm 2.82$, and $40.43 \pm 2.78 \mathrm{D}$ on the second day and 1,3, and 6 months postoperatively, respectively. This result showed a mean flattening of the cornea on the second postoperative day of $3.26 \mathrm{D}$ and a mean flattening of $3.43 \mathrm{D}$ at the end of the follow-up period.The study showed a statistically highly significant difference $(\mathrm{P}<0.001)$.

This coincides with the study by Kanellopoulos et al. ${ }^{(6)}$ who stated that K1 decreased from $44.5 \mathrm{D}$ preoperatively to $42.96 \mathrm{D}$ postoperatively and K2 flattened from $45.3 \mathrm{D}$ preoperatively to 43.96 $\mathrm{D}$ postoperatively, with a mean flattening of the cornea of $2.3 \mathrm{D}$ in the first week postoperatively and $1.44 \mathrm{D}$ after 6 months. Moreover Kanellopoulos et al. 
(6) found that K1 reading decreased from $43.94 \mathrm{D}$ preoperatively to $37.64,37.69,37.66$, and $37.67 \mathrm{D}$ at $1,3,6$, and 12 months postoperatively, whereas $\mathrm{K} 2$ decreased from $45.17 \mathrm{D}$ to $38.32,38.35,38.36$ and $38.37 \mathrm{D}$ at $1,3,6$ and 12 months postoperatively.

\section{CONCLUSIONS}

It could be concluded that the overall goal of all refractive surgery is to get best clear visual acuity and contrast sensitivity. LASIK combined with a prophylactic CXL intervention appears to provide predictability as well as refractive and keratometric stability. The data reported in this study provide evidence of the safety and efficacy of this approach.

The adjuvant CXL procedure adds enhanced corneal biomechanical stability. High myopic and younger age LASIK cases may require biomechanical reenforcement, as means of reducing the incidence and degree of future myopic regression and/or the potential ectasia risk.

\section{REFERENCES}

1. Solomon K, Fernández de Castro L, Sandoval H et al. (2009): LASIK world literature review: quality of life and patient satisfaction. Ophthalmology, 116(4):691-701.

2. Kamaev P, Friedman M, Sherr E et al. (2012): Photochemical kinetics of corneal cross-linking with riboflavin. Invest Ophthalmol Vis Sci., 53(4):23602367.

3. Scarcelli G, Kling S, Quijano E et al. (2013): Brillouin microscopy of collagen crosslinking: noncontact depth-dependent analysis of corneal elastic modulus. Invest Ophthalmol Vis Sci., 54:1418-1425.

4. Chang C, Hersh P. (2014): Corneal collagen crosslinking: a review of 1-year outcomes. Eye Contact Lens, 40(6):345-352.

5. Williams D, Williams G, Porter J et al. (2000): Visual benefit of correcting higher order aberrations of the eye. J Refract Surg., 16(5):S554-9.
6. Kanellopoulos A, Asimellis G, Karabatsas C (2014): Comparison of prophylactic higher fluence corneal cross-linking to control, in myopic LASIK, one year results. Clin Ophthalmol., 8:2373-2381.

7. Reinstein D, Archer T, Srinivasan S et al. (2017): Standard for reporting refractive outcomes of intraocular lens-based refractive surgery. J Refract Surg., 33(4):218-22.

8. Kanellopoulos A (2012): The management of cornea blindness from severe corneal scarring, with the Athens Protocol (transepithelial topography-guided PRK therapeutic remodeling, combined with sameday, collagen cross-linking). Clin Ophthalmol., 6:8790.

9. Kanellopoulos A, Pamel G (2013): Review of current indications for combined very high fluence collagen cross-linking and laser in situ keratomileusis surgery. Indian J Ophthalmol., 61(8): 430-432.

10.Alessio G, L'abbate M, Sborgia $C$ et al. (2013): Photorefractive keratectomy followed by cross-linking versus cross-linking alone for management of progressive keratoconus: two-year follow-up. Am J Ophthalmol., 155:54-65.

11. Celik H, Alagöz N, Yildirim Y et al. (2012): Accelerated corneal crosslinking concurrent with laser in situ keratomileusis. J Cataract Refract Surg., 38:1424-1431.

12. Kymionis G, Grentzelos M, Portaliou D et al. (2014): Corneal collagen cross-linking (CXL) combined with refractive procedures for the treatment of corneal ectatic disorders: CXL plus. J Refract Surg., 30(8):566-76.

13. Reinstein D, Archer T, Randleman J (2013): Mathematical model to compare the relative tensile strength of the cornea after PRK, LASIK, and small incision lenticule extraction. J Refract Surg., 29:454460.

14. Aslanides I, Mukherjee A (2013): Adjuvant corneal crosslinking to prevent hyperopic LASIK regression. Clin Ophthalmol., 7:637-641. 